\title{
A descriptive retrospective cohort study of thoracic surgery experiences from September 2015 to July 2017 at three referral hospitals in Rwanda
}

\author{
Isaie Sibomana', Michael C. Sinclair ${ }^{2,3}$ \\ 1. Department of Surgery, University of Rwanda, Kigali, Rwanda \\ 2. Department of Surgery, University Teaching Hospital of Butare (CHUB), Butare, Rwanda \\ 3. Department of Surgery, University of Virginia, Charlottesville, Virginia, USA \\ Correspondence: Dr Isaie Sibomana (siibomana@gmail.com)
}

(c) 2018 I. Sibomana \& M. Sinclair. This open access article is licensed under a Creative Commons Attribution 4.0 International License (http://creativecommons.org/licenses/by/4.0/), which permits unrestricted use, distribution, and reproduction in any medium, provided you give appropriate credit to the original author(s) and the source, provide a link to the Creative Commons license, and indicate if changes were made.

East Cent Afr J Surg. 2018 Apr;23(1):5-10 https://dx.doi.org/10.4314/ecajs.v23i1.2

\begin{abstract}
Background

Universal access to safe surgery is still a challenge in low- and middle-income countries. An insufficient surgical workforce is a major barrier for performing surgery in these settings, especially specialized operations, such as cardiac and thoracic surgeries. This article describes the thoracic surgical procedures performed at three referral hospitals in Rwanda.

\section{Methods}

We conducted a retrospective cohort study involving patients with various chest pathologies operated by or under the supervision of a thoracic surgeon (faculty from the Human Resources for Health Program), at three teaching hospitals in Rwanda, from September 2015 through July 2017. This study included only major thoracic procedures. Data were collected from the faculty logbook and patient files. The information collected included demographic data, clinical presentation, radiological and intraoperative findings, and outcomes. Ethical approval was obtained from the University of Rwanda College of Medicine and Health Sciences Institutional Review Board.

Results

Thirty-two patients underwent 33 operations during the 23 months of the study ( 1 patient had 2 procedures). Twenty-one of the patients (66\%) were male, and 11 (34\%) were female. Patients' ages ranged between 13 and 77 years, with a mean age of 41 years. Infectious chest pathologies (mostly tuberculosis-related) were common indications for surgery. Sixteen cases (48\%) were thoracic empyemas that required either thoracotomy and pulmonary decortication or open thoracostomy (modified Eloesser flap). Other operations performed were anterior mediastinotomy for mediastinal mass (4 cases), biopsy and resection of chest wall mass (3 cases), pericardial window for pericardial tamponade (2 cases), resection of lung aspergilloma (2 cases), resection of a lung tumour ( 2 cases), and others (4 cases). Mortality was $6 \%$ ( 2 patients), and 3 patients had postoperative complications, which were surgical site infection in 1 patient and ineffective thoracotomy in 2 patients.
\end{abstract}

\section{Conclusions}

With clinical mentorship and dedicated teams, thoracic surgery can be performed in low-resource settings, where infectious pathologies predominate, with acceptable morbidity and mortality.

Keywords: thoracic surgery, thoracostomy, tuberculosis, empyema, outcomes, complications, Rwanda

\section{Introduction}

There are obstacles to performing thoracic surgery in parts of the world where there are limited resources. The challenges and limitations include those related to preoperative diagnostic imaging, specific anaesthetic considerations, surgical instrumentation, postoperative management, and surgical technical skills.

Rwanda is in the midst of the implementation of major innovation in healthcare education and delivery. As part of this ambitious endeavour, the Human Resources for Health, 
Table 1. Thoracic surgery cases during the study period

\begin{tabular}{l|c}
\multicolumn{1}{c|}{ Operation } & $\mathbf{n}$ \\
\hline \hline Pulmonary decortication & 10 \\
Open thoracostomy & 6 \\
Anterior mediastinotomy & 4 \\
Biopsy/resection chest wall tumour & 3 \\
Resection of aspergilloma & 2 \\
Pericardial window & 2 \\
Resection of lung neoplasm & 2 \\
Pericardiectomy & 1 \\
Other & 3 \\
\hline
\end{tabular}

Rwanda Program (HRH) brought faculty from over 20 medical institutions in the United States to work in health facilities in Rwanda starting from 2012. During a 2-year period, 1 of the authors of this article (MCS), who is an American thoracic surgeon, worked at teaching hospitals in Rwanda as $\mathrm{HRH}$ faculty, and many thoracic surgical procedures were performed, and local specialists were trained. ${ }^{1}$

This article describes thoracic surgical procedures performed in 23 months at 3 referral hospitals in Rwanda: University Teaching Hospital of Butare (CHUB), University Teaching Hospital of Kigali (CHUK), and King Faisal Hospital (KFH), under the supervision of MCS.

\section{Methods}

A retrospective cohort study was conducted at 3 referral hospitals in Rwanda over 23 months (1 September 2015 through 31 July 2017). The main objectives were to describe the thoracic surgical procedures performed and the barriers and challenges faced. Major thoracic surgical cases were identified from the surgical logbook of the faculty thoracic surgeon. Thoracic surgical procedures that involved the musculoskeletal components of the chest wall or required opening the chest, conducted under general anaesthesia, were considered "major" and were included in this series. Additional clinical information was obtained from patient hospital records. The number of chest operations done by other short-term visiting surgeons or local general surgeons during this period is unknown, and these were excluded. We also excluded minor thoracic procedures (those performed under local anaesthesia) and chest operations performed by paediatric surgeons. Ethical approval for this study was obtained from the University of Rwanda College of Medicine and Health Sciences Institutional Review Board.

\section{Results}

During this 23-month interval, 32 patients underwent 33 thoracic surgical procedures (Table 1). Seventy per cent of the operations were performed by surgical residents or junior faculty members under the supervision of the thoracic

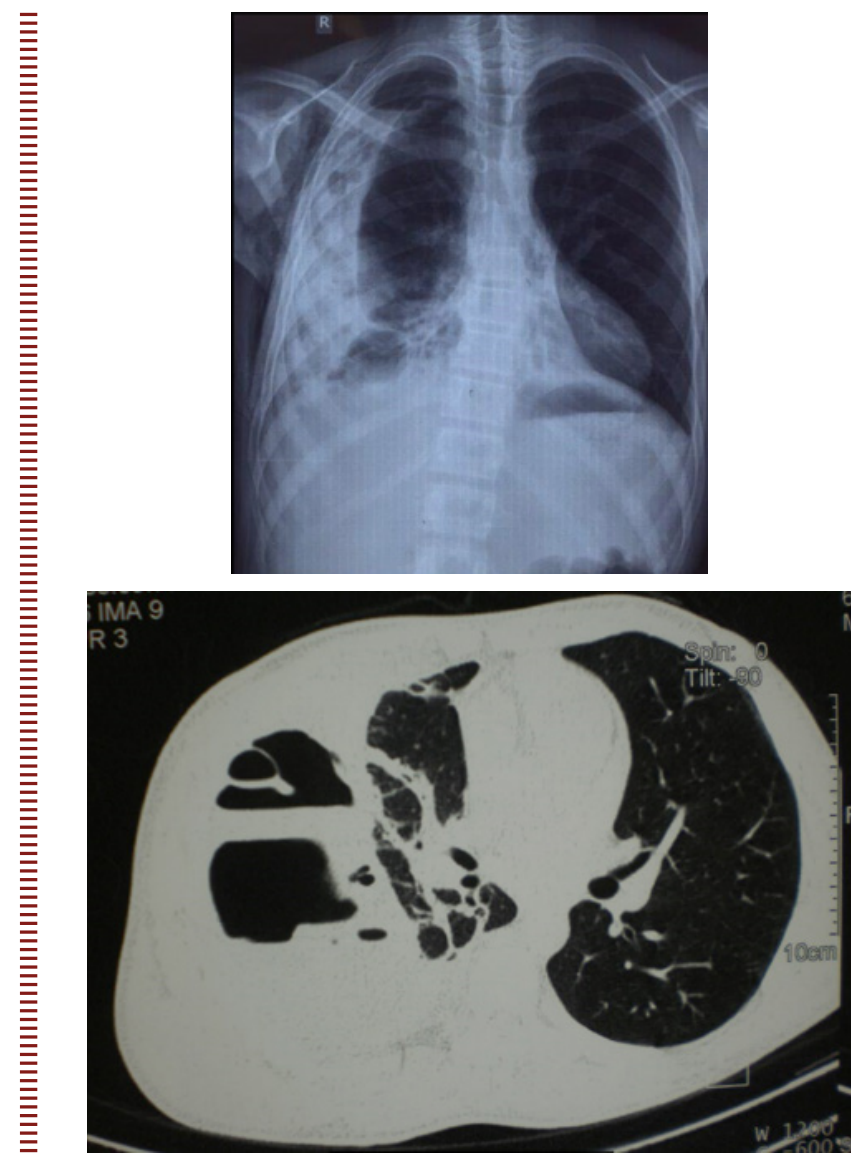

Figure 1. Chest radiograph and CT scan of typical patient with empyema thoracis with thickened pleural peel and pyogenic debris prior to pulmonary decortication

surgeon. Occasionally the thoracic surgeon was the primary operating surgeon. Twenty-eight of the operations (85\%) were performed at CHUB.

Preoperative evaluations consisted of routine chest $\mathrm{x}$-rays, full-blood counts, and electrolyte measurements. As most of the operations were performed at CHUB, which did not have a computed tomography (CT) scanner during the study period, there were only a few CT images obtained at another referral hospital. Therefore, the number of patients who underwent preoperative chest CT scanning was limited. Only 2 patients underwent preoperative bronchoscopy-1 had massive haemoptysis from an apical pulmonary cavitary lesion that contained an aspergilloma, and 1 had a post-traumatic tracheo-oesophageal fistula (TOF). The bronchoscopy on the patient with haemoptysis showed no abnormalities. The patient with the TOF will be discussed in detail below. Pulmonary function tests, cardiac stress tests, and arterial blood gas measurements were not available. The exact number of microbiologic examinations of the pleural exudates and histological evaluations of specimens of patients with apparent infectious processes are not known. If a malignant process was suspected, an operative specimen was sent for histological examination.

All operations were performed with a single-lumen endotracheal tube. Eighty-eight per cent of patients were extubated in the operating room at the conclusion of the proce- 

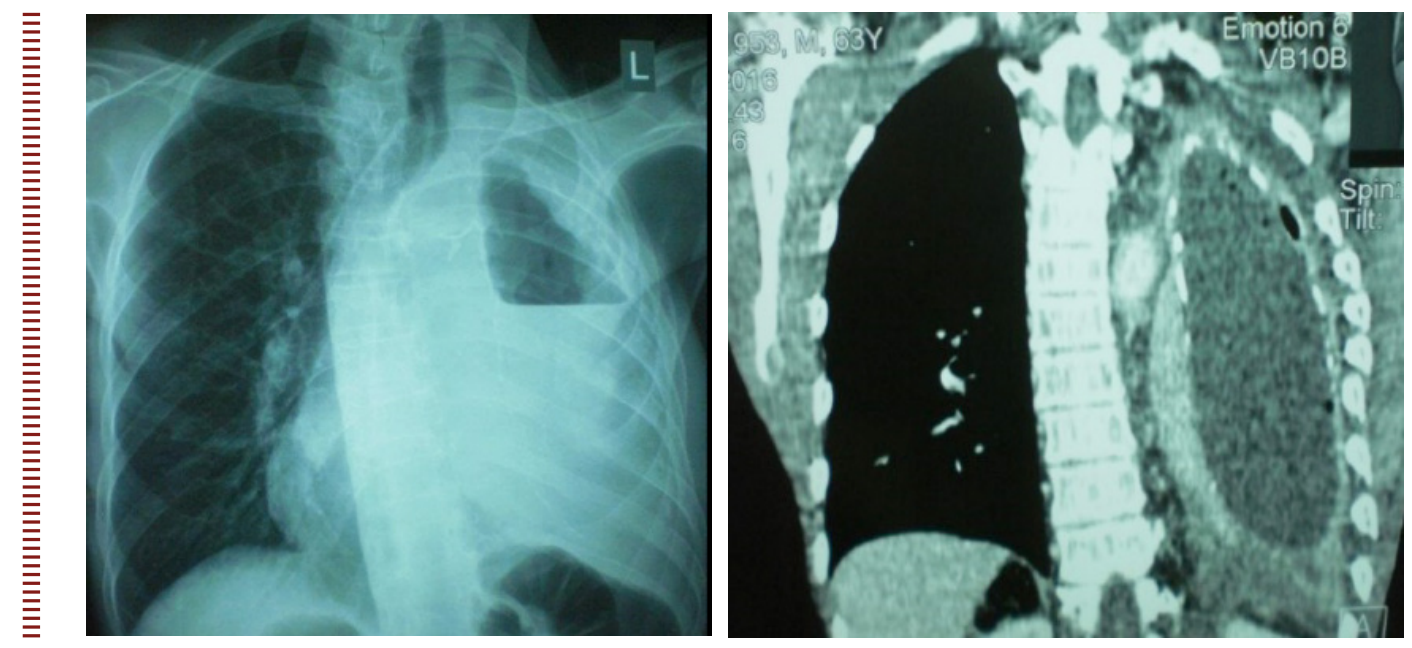

Figure 2. Chest x-ray and computed tomography images of empyema thoracis prior to modified Eloesser flap
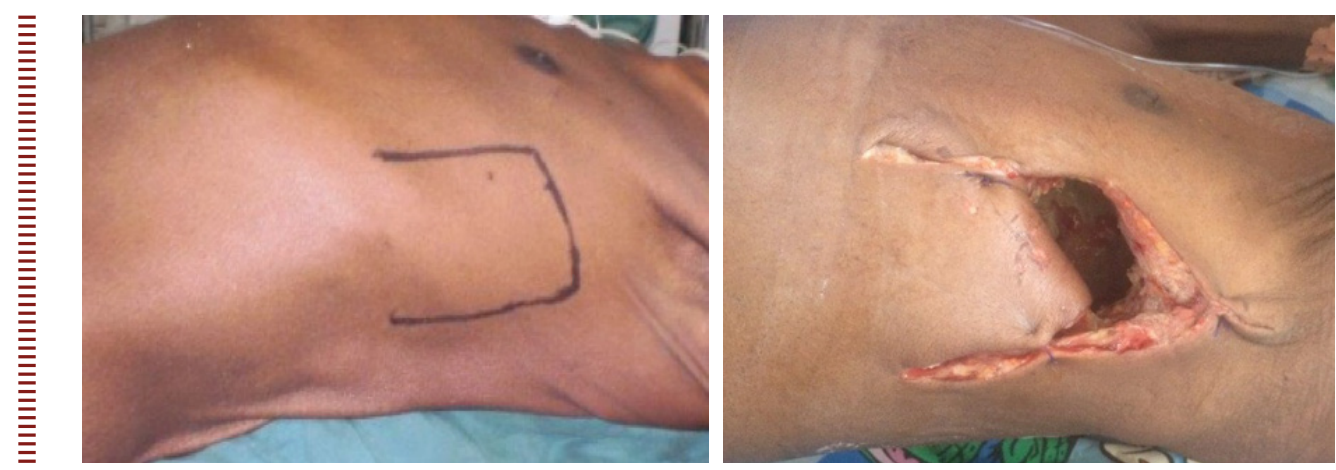

Figure 3. Open thoracostomy (modified Eloesser flap) - preoperative (with incision outline) and postoperative

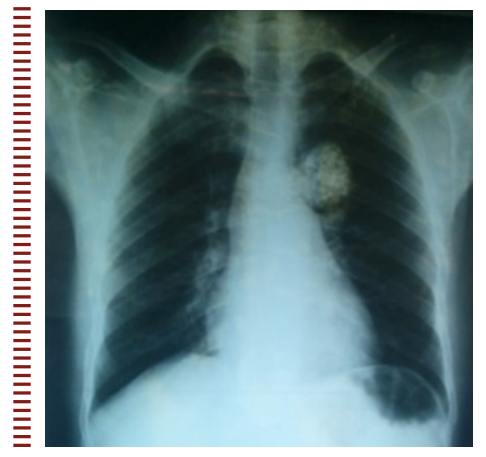

Figure 4. Chest x-ray of patient with hamartoma

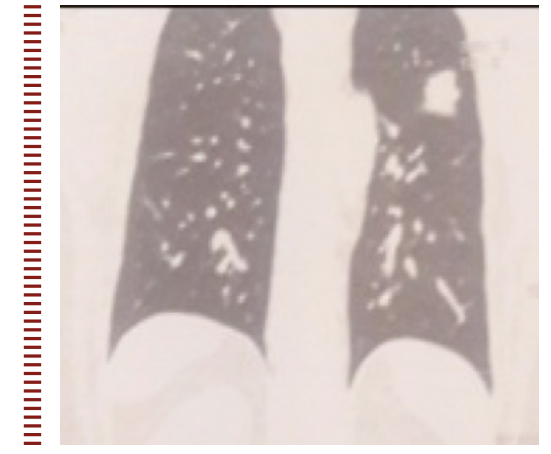

Figure 5. Computed tomography scan - left upper lobe adenocarcinoma dure. One patient (pericardiectomy) was haemodynamically unstable at the end of the operation and was taken to the Intensive Care Unit (ICU) without being extubated. Three additional patients, each haemodynamically stable with adequate oxygen saturation, were sent from the operating room to the ICU intubated because of disagreement or miscommunication between the surgical team and the anaesthesiologists.

Ten of the 33 cases (30\%) were pulmonary decortications. All but 1 of the decortications was performed to allow expansion of a lung compressed by a thick visceral pleural peel and pyogenic debris that could not be evacuated using chest tubes (Figure 1). Ninety-three per cent of purulent col- lections were related to parapneumonic effusions or suspected tuberculous empyemas that had become secondarily infected. One patient had an infected haemothorax caused by blunt chest trauma. Another patient underwent decortication to expand a lung trapped by a partially calcified chronic fibrothorax.

An open thoracostomy (modified Eloesser flap) was performed in 5 patients. One of these patients returned several months later because the opening into the pleural space had become narrowed by scar contracture; the thoracostomy was satisfactorily revised. All 5 patients with thoracostomies had pyogenic empyemas thought to be secondary to tuberculous empyemas that had become secondarily infected with pyogenic organisms (Figures 2 and 3 ).

After control of sepsis, the thoracostomy was obliterated in 1 patient using a latissimus dorsi flap. The remaining 4 patients had good tolerance to thoracostomy and were discharged to continue local wound care and cavity irrigation at their respective home district hospitals.

Both patients with aspergillomas had a prior history of treated pulmonary tuberculosis. Both had residual apical cavities containing opacities consistent with a fungus ball, and both had had massive, life-threatening haemoptysis. In 
Table 2. Patient demographics and outcomes of thoracic surgery cases during the study period

\begin{tabular}{l||c}
\hline Age (years) & $13-77$ (mean 41) \\
Sex & Male 21 (66\%) \\
Female 11 (34\%) \\
Deaths & $2(6 \%)$ \\
Intraoperative complications & 0 \\
Re-explorations & 0 \\
Postoperative complications & $3(9 \%)$ \\
\hline
\end{tabular}

both cases, the apex of the affected lung containing the fungus ball was resected.

Four anterior mediastinotomies (Chamberlain procedure) were performed in patients who had mediastinal masses that were thought to be malignant but unresectable.

Two patients with cardiac tamponade underwent pericardial window operations. One of the patients had uraemic pericarditis; the other had recently undergone mitral and tricuspid valve surgery and had a large pericardial effusion refractory to repeat pericardiocentesis.

Two patients underwent thoracotomy and resection of lung neoplasms; 1 was benign (Figure 4), and the other was malignant (Figure 5).

One patient underwent pericardiectomy for acute constrictive pericarditis thought to be secondary to recent blunt chest trauma.

All decortications, both pulmonary resections for aspergillomas, and both resections of lung tumours were done via posterolateral thoracotomies entering the chest through either the fifth intercostal space or the bed of the resected fifth rib. Multiple intercostal blocks with a long-acting local anaesthetic (bupivacaine) were performed before chest closure in these cases.

The anterior mediastinotomies were performed with the patients in the supine position with the operative side slightly elevated. An anterior costal cartilage and a small part of the corresponding rib were resected over a prominent part of the mass adjacent to the anterior chest wall to provide access for biopsies.

Both pericardial windows and the pericardiectomy were done through left anterolateral thoracotomies in the fourth or fifth intercostal space with the left side of the chest slightly elevated.

The results are summarized in Table 2.

The age range of the patients was 13 to 77 years with a mean of 41 years. Twenty-one of the patients were male (66\%), and eleven were female (34\%).

\section{Deaths}

Two patients died. One was a 77-year-old man who was the first patient to undergo pulmonary decortication at that institution. Despite a preoperative discussion and plan to ex- tubate the patient in the operating room, he was taken to the ICU intubated. He was left intubated and sedated for 1 week after which he failed extubation.

The other death occurred in a 36-year-old woman with a large bilateral anterior mediastinal mass, a left pleural effusion, and partial collapse of the left lung. She was dependent on supplemental oxygen preoperatively. She was initially extubated but was reintubated when she developed a small postoperative pneumothorax on the operated (right) side. Despite bilateral chest tube placement and reintubation, she had progressive respiratory decompensation, which led to her death.

\section{Complications}

There were no intraoperative complications and no patients required return to the operating room for re-exploration. Three patients developed postoperative complications.

A 48-year-old woman who underwent resection of a right apical lung cavity containing an aspergilloma developed a surgical site infection involving the skin, subcutaneous tissue, and superficial muscle layers. The infection did not extend into the intercostal muscles or pleural cavity. She was treated with antibiotics, dressing changes, and local wound debridement on the ward and the wound healed satisfactorily.

A pulmonary decortication was done on a 48 -year-old man, but the lung failed to expand. He had a pyogenic empyema thought to have been due to a tuberculous empyema that had become secondarily infected. The postoperative course was uneventful, but the chest x-ray was unchanged. He was seen several months later, and his $\mathrm{x}$-ray was identical to the preoperative one. His moderate exertional dyspnoea was likewise unchanged.

A 32-year-old woman suffered a stab wound at the base of the neck with near complete transection of the trachea. The trachea was repaired through the stab wound, but at the time of the repair, the oesophagus was not visualized. Approximately 1-week post-injury, she developed symptoms of TOF (aspiration associated with swallowing). She underwent bronchoscopy and oesophagoscopy, and the fistula was reported to be $3 \mathrm{~cm}$ above the carina. Because of the reported location of the fistula, a right thoracotomy was performed, and the trachea was mobilized from the carina to $8 \mathrm{~cm}$ proximally. No fistula was identified, but a patch of azygos vein and pleura was placed between the trachea and oesophagus. After the thoracotomy, the patient's symptoms persisted. Repeat bronchoscopy and oesophagoscopy correctly located the fistula at the thoracic inlet on the left. The fistula was satisfactorily repaired at a subsequent operation using a cervical approach.

\section{Other}

Pott's paraplegia continues to be a vexing problem in much of the developing world. One patient presented with kyphosis and worsening paraplegia for 4 months despite antituberculous chemotherapy. Imaging showed destroyed T1T2 vertebrae with abscess collection (Figure 6). Although 


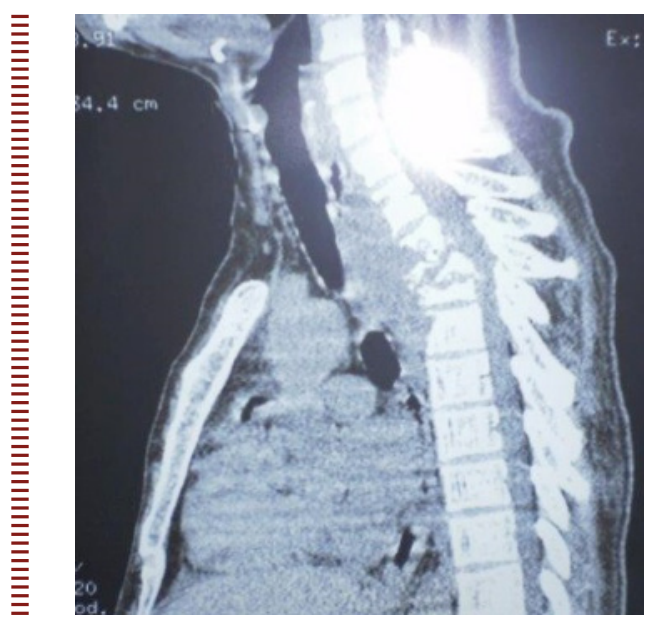

Figure 6. Cervicothoracic computed tomography image of a patient with Pott's disease and abscess collection

many patients with paraplegia secondary to tuberculosis of the spine can be satisfactorily managed by antituberculous medication alone, some patients require operative intervention to drain the abscess and decompress the spinal cord. Costotransversectomy is an old but still useful procedure, provided it is done for the appropriate patients. ${ }^{2}$

\section{Discussion}

Tuberculosis is the leading cause of empyema which leads to thoracic surgical procedures in developing countries. ${ }^{3}$ Current drug regimens achieve a cure rate $>85 \%$ in patients with pulmonary tuberculosis, but there are less satisfactory results in geographical areas where multidrug-resistant (MDR) strains are prevalent. The need for surgery is estimated to have increased from $5 \%$ to $15 \%$ over the last 20 years owing to the growing emergence of MDR-TB. ${ }^{4}$

In this study, intrathoracic infections were the indication for more than half of the procedures (10 decortications, 6 open thoracostomies, and 2 aspergillomas). Patients with empyemas had a history of tuberculosis treatment plus several attempts at chest tube drainage that failed to remove the pus. Ideally, patients with empyema need surgery for drainage of chronic pleural infection and to allow lung expansion. Decortication is the best and only available choice in resource-limited settings. The decision for decortication mainly depends on patient performance status, nutritional status, and ability to tolerate an extended period of general anaesthesia.

Although pulmonary decortication is more likely to provide good pulmonary function as well as removal of the septic focus, some of our patients were severely debilitated and malnourished. It was felt that these individuals would not tolerate decortications. For such patients, open thoracostomy as first described by Eloesser in 1935 (but subsequently modified by many authors), is a reasonable alternative. ${ }^{5-7}$ These patients are usually left with a permanent open chest wound, but it is possible for them to lead a relatively normal life, as they are unlikely to be subjected to repeated bouts of sepsis or chronic infection. Furthermore, after the sepsis is controlled, if the patient's general health status improves, it is possible for the cavity to be obliterated using muscle flaps. ${ }^{8}$

Unfortunately, 2 of the 32 patients (6\%) died. It is not clear whether the patient with the decortication who died would have survived if the original plan (extubation in the operating theatre) had been carried out. It is possible that he was simply too old and debilitated for such an operation. ${ }^{9}$ However, the adverse outcome, in this case, underscores the need for better communication between the surgeons and anesthesiologists. The other death was probably unavoidable, and perhaps the biopsy should not have been offered to this critically ill patient.

Complications are of foremost concern in thoracic surgery. Infection of thoracotomy incisions is uncommon, and the wound infection, in this case, was probably secondary to a break in aseptic technique, as it was unlikely to be related to the pulmonary fungus infection.

The failure of the lung to expand after an apparently satisfactory pulmonary decortication suggests that the underlying lung parenchyma was fibrotic preventing the lung from expanding. Had this been recognized preoperatively (for example, by CT scan), it would have been better to perform an open thoracostomy or avoid surgery altogether.

The inability to find the TOF was the result of a management error. It was not reasonable to expect that the traumatic TOF caused by a stab wound to the neck would be located so far distally as to necessitate a right thoracotomy. The surgical team should have questioned the endoscopy report and thus avoided an unnecessary thoracotomy.

Two other cases merit further discussion. The patient with acute constrictive pericarditis might have been better served with a median sternotomy, which was not available in our setting. On the other hand, it was possible to perform a satisfactory cardiac decortication in this instance through a left anterior thoracotomy.

One patient underwent thoracotomy to repair a rupture of the left hemidiaphragm secondary to blunt trauma. Rupture of the diaphragm should generally be repaired by laparotomy. However, if the diagnosis is made late (in this case several months after the incident), it is preferable to perform a thoracotomy to facilitate the division of adhesions between the herniated viscera and the lung or mediastinum. In this patient, half of the left hemithorax was occupied by the herniated, dilated gastric fundus.

Although universal access to safe surgery is still a challenge in low- and middle-income countries, this study indicates that some specialized surgeries, such as cardiac and thoracic can still be performed in these settings to treat infection-related morbidities with satisfactory outcomes.

\section{Conclusions}

Infectious diseases continue to be a source of major morbidity and mortality in Rwanda. Chest infections that could not be treated adequately with antibiotics alone accounted for more than half of the patients who required surgical intervention in this series. The increasing incidence of MDRTB 
contributes to this problem. This modest experience in Rwanda suggests that thoracic surgery has a role in the management of some patients in resource-limited settings. To meet this need, thoracic surgeons need to be trained, and support services (including anaesthesiology, diagnostic imaging, and laboratory capabilities) must be enhanced.

\section{Competing interests}

Both authors declare that they have no competing interests related to this work.

\section{References}

1. Republic of Rwanda Ministry of Health Human resources for health strategic plan 2011-2016 [Internet]. Kigali: Government of Rwanda; 2011. Available from: http:// www.moh.gov.rw/fileadmin/templates/ policies/HRH_STRATEGIC_PLAN_2011_2016_APPROVED.pdf

2. Botha $\mathrm{AH}$, Davis JH. Costotransversectomy in thoracic spinal tuberculosis. SA Orthop J. 2016 Apr;15(1):83-6. doi: 10.17159/23098309/2016/v15n1a10

3. Khan YA. Bilateral suspected tuberculous empyema thoracis. APSP J Case Rep. 2012 May;3(2):13. Epub 2012 Jun 1.

4. Subotic D, Yablonskiy $P$, Sulis $G$, et al. Surgery and pleuro-pulmonary tuberculosis: a scientific literature review. J Thorac Dis. 2016 Jul;8(7):E474-85. doi: 10.21037/jtd.2016.05.59.
5. Eloesser L. An operation for tuberculous empyema. Dis Chest. 1935 Oct;1(8):8-9. doi: 10.1378/chest.1.8.8.

6. Thourani VH, Lancaster RT, Mansour KA Miller $\mathrm{J} \mathrm{J}$ r. Twenty-six years of experience with the modified Eloesser flap. Ann Thorac Surg. 2003 Aug;76(2):401-5 discussion 405-6. doi: 10.1016/S00034975(03)00470-3

7. Symbas PN, Nugent JT, Abbott OA, Logan WD Jr, Hatcher CR Jr. Nontuberculous pleural empyema in adults. The role of a modified Eloesser procedure in its management. Ann Thorac Surg. 1971 Jul;12(1):69-78. doi :10.1016/S00034975(10)65095-3.
8. García-Yuste M, Ramos G, Duque JL, et al. Open-window thoracostomy and thoracomyoplasty to manage chronic pleural empyema. Ann Thorac Surg. 1998 Mar;65(3):818-22. doi: 10.1016/S00034975(97)01386-6

9. Marks DJ, Fisk MD, Koo CY, et al. Thoracic empyema: a 12-year study from a UK tertiary cardiothoracic referral centre PLoS One. 2012;7(1):e30074. doi: 10.1371/ journal.pone.0030074. Epub 2012 Jan 20. 